\title{
ESTUDIO ELECTROFISIOLOGICO DE LAS VARIACIONES EN LA INERVACION DE LOS MUSCULOS DE LA MANO
}

\author{
R. C. REY* \\ OLGA P. SANZ ** \\ R. E. P. $S I C A * *$
}

Las variaciones en la inervación de los músculos intrínsecos de la mano 1,13,14,16, así como la anastomosis entre los nervios mediano y cubital 3,10,14,15,20, son con frecuencia fuentes de error en la interpretación de estudios de velocidad de conducción nerviosa 2,4,7,11, pudiendo representar escollos en el diagnóstico de las affecciones de dichos nervios.

El motivo de la presente comunicación es tratar de delinear una metodología sencilla y evaluar el probable comportamiento en distintas patologías, de las variaciones halladas.

\section{MATERIAL $\mathbf{Y}$ METODO}

Se investigaron 24 manos en 12 voluntarios sanos (6 hombres y 6 mujeres), cuyas edades oscilaron entre los 25 y 50 años.

Un estimulador bipolar, fue empleado en forma percutánea para la estimulación de los nervios mediano y cubital a nivel del codo y de la muñeca. La duración del estímulo fue de $100 \mu \mathrm{s}$ y su intensidad máxima, cuidando que el campo eléctrico generado por la estimulación, no indujera actividad en el otro nervio. Con electrodo de superficie esférico de platíno de $1 \mathrm{~mm}$ de diámetro, se registró en abductor pollicis brevis (APB), adductor digiti minimi (ADM), y en el primer interóseo dorsal (PID); tomándose como referencia electrodos de cobre colocados en meñique $e$ índice, durante los estímulos del mediano y cubital, respectivamente. Un electrodo, laminar, de plata, ubicado en el dorso de la mano, fue utilizado como tierra (fig. 1). Con el electrodo de registro se investigó cada músculo hasta obtener um potencial cuya primera deflexión fuese de polaridad negativa, estimándolo proveniente de la zona de placas motoras, descartando los otros potenciales por considerarlos de campo alejado. Una vez hallado el potencial de la zona de placas motoras se midieron su amplitud y duración. Con la metodología descripta se exploraron sucesivamente los músculos APB, ADM y PID, durante la estimulación de: cubital en codo; cubital en muñeca; mediano en codo; mediano en muñeca.

Trabajo de la Sección de Electronuerofisiología Clínica, División Neurologia, Hospital Ramos Mejía, Buenos Aires, Argentina: * Médico Residente; ** Médico Assistente; *** Jefe de Sección. 


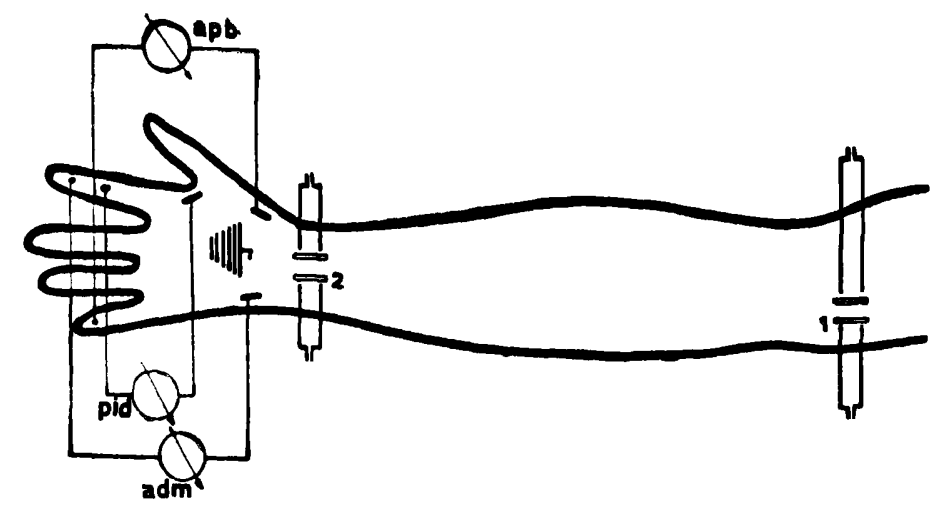

Hig. 1 - Esquema de la técnica empleada. $A P B$ - abductor pollicis brevis, $A D M$ - adductor digiti minimi, PID - primer interoseo dorsal.
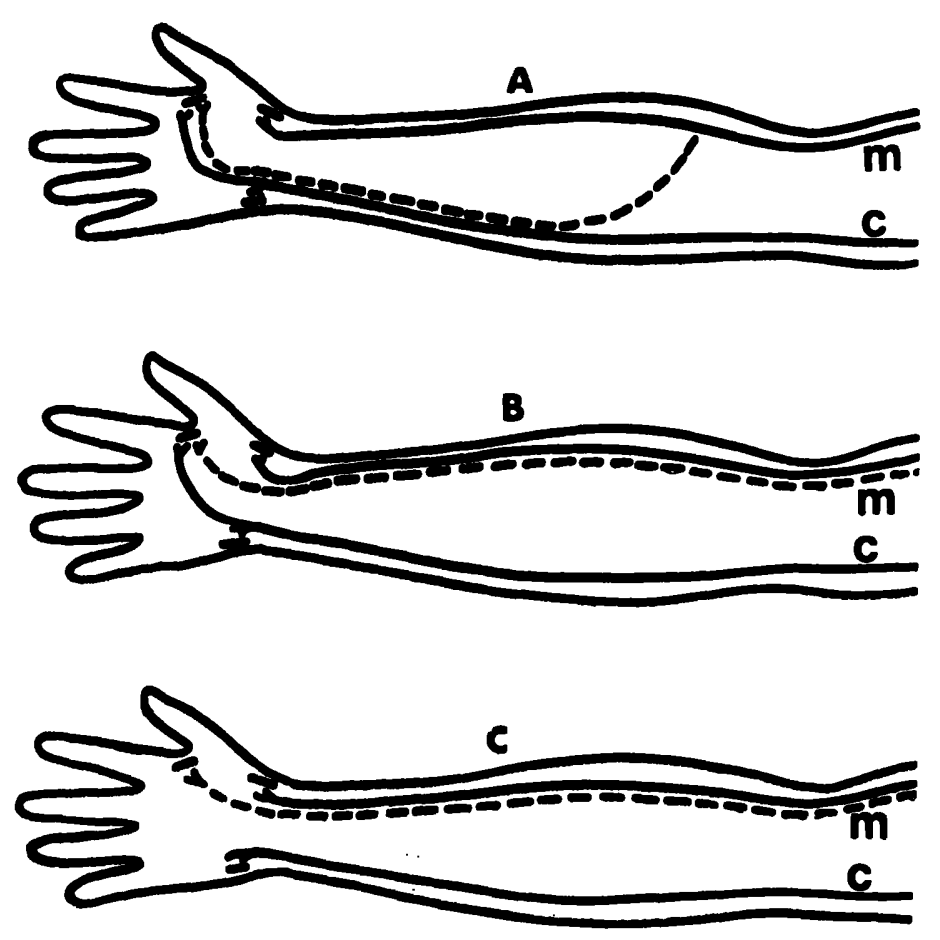

Fig. \& - Variaciomes en la inervación de los musculos de la mano por parte de los nervios mediano y cubital. $M$ nervio mediano, $C$ - nervio cubital.

\section{RESULTADOS}

Los resultados obtenidos se resumen en la tabla 1. En 11 manos se obtuvó potencial en PID durante el estímulo de mediano en codo. En 4 de estas no se registró durante su estimulación en muñeca. Este hallazgo indicaría la presencia de fibras motoras que se dirigirian del mediano al cubital en el antebrazo, hacia el PID (Fig. 2a). La importancia del aporte del mediano osciló entre el 1,63 y el $41,1 \%$ del potencial, con una media del 19,41\%. Las otras 7 manos donde se registró potencial en PID durante el estímulo del mediano en codo $y$ en mufeca, indicarian la presencia de fibras motoras del mediano que se anastomosarian con el cubital en palma o se dirigirian directamente al PID. En 6 de estas manos la inervación del PID era compartida con el cubital (Fig. 2b), siendo la importancia del aporte del mediano de un 6,2 a un $15,6 \%$ del potencial, con una media del 10,35\%. En la mano restante, el PID era inervado en un $100 \%$ por el mediano, no registrándose aporte del cubital (Fig. 2c). 
16 ARQ. NEURO-PSIQUIATRIA (SAO PAULO) VOL. 42, No 1, MARGO,

1984

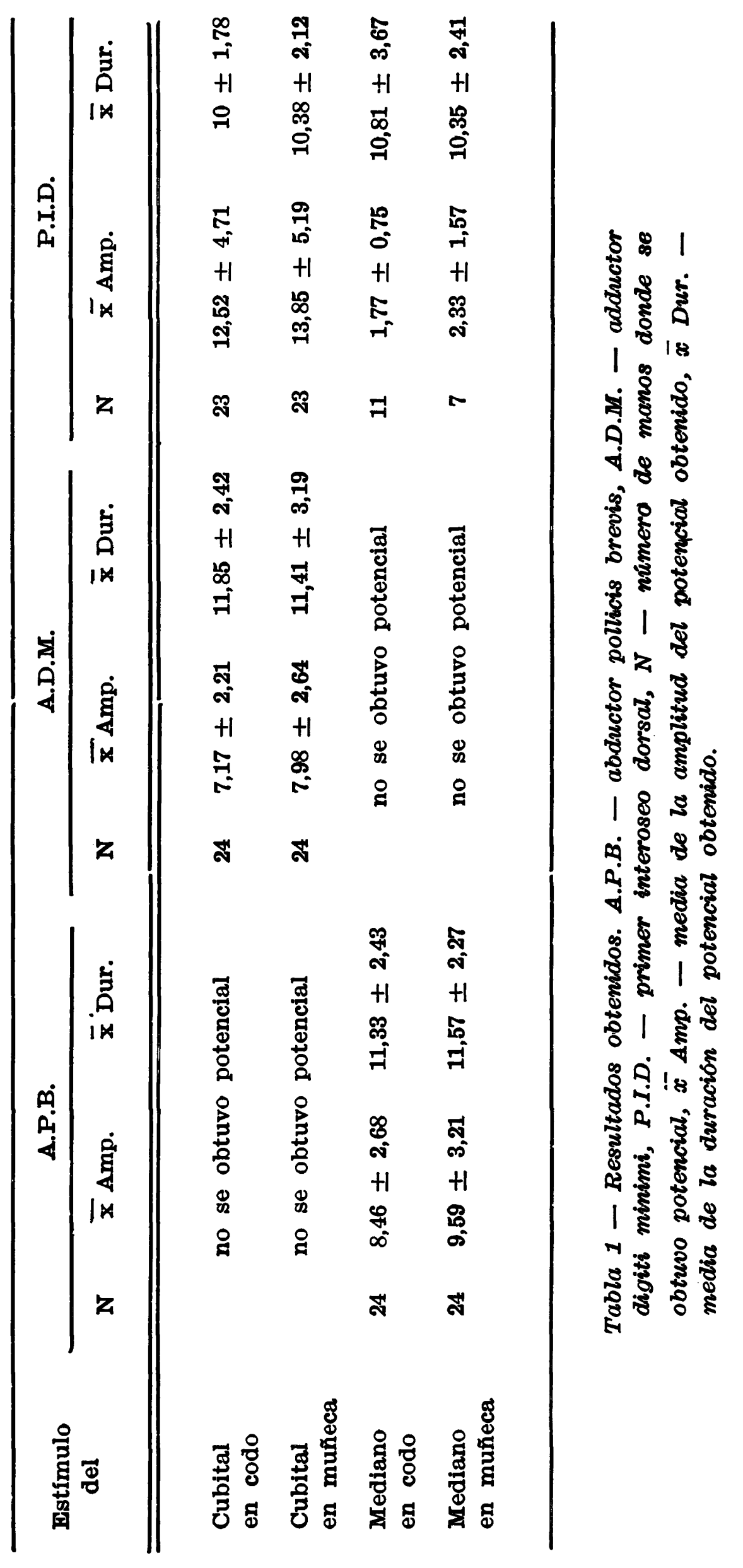




\section{COMENTARIOS}

El registro del potencial evocado muscular durante la estimulación de un nervio, plantea dos posibilidades: que el potencial recogido sea conducido por volúmen, generado en músculos distantes inervados por el nervio estimulado; que este envíe fibras al músculo y por lo tanto el potencial registrado provenga del músculo explorado 8,11. En la metodología descripta sólo se consideraron los potenciales provenientes de la zona de placas motoras, asegurando que el potencial registrado provenía del músculo explorado y no de músculos distantes. La anastomosis de Martin-Gruber ${ }^{3,15}$, lleva fibras motoras del mediano al cubital en el antebrazo, destinadas al PID y menos frecuentemente a los músculos de la eminencia tenar y/o hipotenar 12,19. Cuatro de las manos exploradas presentaron esta anomalía; la incidencia encontrada, $1666 \%$, es similar a la referida en la literatura $3,5,12$. En caso de afectación del mediano en codo, en alguno de estos sujetos, ésta podría presentar compromiso del PID, cuya importancia dependería de la cantidad de fibras que aportase el mediano. Así mismo, una lesión del cubital en codo produciría menor compromiso del PID, que si la lesión estuviese en muñeca.

En siete manos $(29,16 \%)$ el mediano aportaba fibras al PID en forma directa o por anastomosis con el cubital en palma, este hecho es mencionado por otros autores 13,17,18, sin aportar datos cuantitativos. En una de estas manos el PID era inervado exclusivamente por el mediano, la afectación del mismo en codo o en muñeca, agregaría a los hallazgos habituales, el compromiso del PID y una lesión del cubital cursaría con indemnidad del mismo. No se hallaron fibras del cubital dirigiéndose al mediano, hecho de por sí poco habitual 10,13,20. Por último queremos enfatizar, la frecuente inervación del PID por el mediano (11 manos, 45,83\%), mediante una anastomosis con el cubital en el antebrazo y, más frecuentemente aún, por una anastomosis en palma 0 en forma directa. Si bien, la técnica descripta no permite investigar probables anastomosis sensitivas, la consideramos de aplicación más sencilla e inocua que las propuestas anteriormente $2,6,9,12,21$.

\section{RESUMEN}

Para investigar las probables variaciones en la inervación de los músculos intrínsecos de la mano y/o la anastomosis entre los nervios mediano y cubital, se estudiaron 24 manos, estimulando en forma percutánea a dichos nervios, registrándose en abductor pollicis brevis (APB), adductor digiti minimi (ADM) y primer interóseo dorsal (PID). Se halló una frecuente inervación del PID por el mediano. Se describe la técnica utilizada, comentando incidência, importancia y probable comportamiento en distintas patologías de las variaciones halladas. 
SUMMARY

Electrophysiological study of the variations in the innervation of the muscles of the hand.

An electrophysiological study was carried out searching for variations in the innervation of the muscles of the hand. For this purpose a simple and non time-consuming technique was designed for stimulating the ulnar and median nerves at the elbow and wrist. Recordings were performed with surface electrodes at the abductor pollicis brevis, adductor digiti minimi and first dorsal interosseus muscles. Several anomalous innervation patterns of the intrinsic muscles of the hand were found, and the innervation of the first dorsal interosseus by the median nerve was the most frequent.

\section{REFERENCIAS}

1. BROOKS, H. - Variations in nerve supply of Flexor Brevis Pollicis. Anat. Physiol. 20:641, 1886.

2. GASSEL, L. - Sources of error motor nerve conduction studies. Neurolgy (Minneapolis) 14:825, 1964.

3. GRUBER, W. - Uber die Verbidung des Nervus medianus mit dem Nervus ulnaris am Unterarm des Saugethiere. Arch. anat. physiol. Wissensch. med. 37: 501, 1870 .

4. GUTMAN, L. - Median-Ulnar nerve communications and carpal tunnel syndrome. J. Neurol. Neurosurg. Psychiat. 40:982, 1977.

5. HIRASAWA, K. - Untersuchungen uber das periphere Nervensystem Plexus brachialis und die Nerven der obern Extremitat. Arb. Anat. Inst. Kaiserlichen Univ. Kyoto. 2:135, 1931.

6. HOPF, H. \& HENSE, W. - Anomalien der motorischen Innervation an der Hand. Flectroencephalogr. Electromyogr. 5:220, 1974.

7. IYER, V. \& FENICHEL, G. - Normal median nerve proximal latency in carpal tunnel syndrome: a clue to coexisting Martin-Gruber anastomosis. J. Neurol. Neurosurg. Psychiat. 39:449, 1976.

8. KIMURA, J. - Collision technique: physiological block of nerve impulses in studies of motor nerve conduction velocity. Neurology (Minneapolis) 26:680, 1976.

9. KIMURA, J.; MURPHY, M. \& VARDA, D. - Electrophysiological study of anomalous innervation of intrinsic hand muscles. Arch. Neurol. (Chicago) 33: 842, 1976.

10. KOMAR, J.; SZEGVARI, M. \& GLOVICZKY, Z. - Traumatischer Durchschanitt des N. medianus ohne komplette motorische Parese: Martin-Gruber Anastomose. Nervenarzt 49:697, 1978.

11. LAMBERT, E. - Diagnostic value of electrical stimulation of motor nerves. Electroencephalogr. clin. Neurophysiol. 22 (suppl):9, 1962.

12. MANNERFELT, L. - Studies on the hand in ulnar nerve paralysis: a clinical experimental investigation in normal and anormalous innervation. Acta orthop. scand. 87 (suppl):23, 1966.

13. MARINACCI, A. - The problem of unusual anomalous innervation of hand muscies: the value of electrodiagnosis in its evaluation. Bull. Los Angeles neurol. Soc. 29:133, 1964.

14. MARINACCI, A. \& VON HAGHN, K. - Misleading «all median hand». Arch. Neurol. (Chicago) 12:80, 1965.

15. MARTIN, F. - Tal om Nervers Allmanna Engenskaper i Manniskans Kropp. L. Salvius, Stockholm, 1763. 
16. MURPHEY, F.; KIRKLIN, J. \& FINLAYSON, A. - Anomalous inervation of the intrinsic muscles of the hand. Surg. Gynecol. Obst. 83:15, 1946.

17. POLLOCK, L. DAVIS, L. - Peripheral Nerve Injuries. Paul B. Hoeber Inc., New York, 1932.

18. ROWNTREE, T. - Anomalous innervation of hand muscles. J. Bone Joint Surg. 31B:505, 1949.

19. SRINIVASAN, R. \& RHODES, J. - The median-ulnar anastomosis (Martin-Gruber) in normal and congenitally abnormal fetuses. Arch. Neurol. (Chicago): 38:418, 1981.

20. STREIB, W. - Ulnar-to-median nerve anastomosis in the forearm: electromyographic studies. Neurology (Minneapolis) 29:1534, 1979.

21. WILBOURN, A. \& LAMBERT, E. - The forearm median to ulnar nerve communication: electrodiagnostic aspects. (Abstract). Neurology (Minneapolis): 26: 368, 1976.

Sección de Electroneurofisiología Clinica, Division Neurología, Hospital Ramos Mejia - Urquiza 609 - 1221 Buenos Aires - Argentina. 